EPJ Web of Conferences 62, 07003 (2013)

DOI: $10.1051 /$ epjconf/20136207003

(C) Owned by the authors, published by EDP Sciences, 2013

\title{
The even-odd effect in fission-fragment $Z$ yields - a new kind of nuclear clock
}

\author{
Beatriz Jurado a and Karl-Heinz Schmidt \\ CENBG, CNRS/IN2P3, Chemin du Solarium, BP. 120, 33175 Gradignan, France
}

\begin{abstract}
A model for the even-odd effect in fission based on statistical mechanics is presented. It reveals that the variation of the even-odd effect with the mass of the fissioning nucleus and the increase towards asymmetric splits is due to the important statistical weight of configurations where the light fission fragment populates the ground state of an eveneven nucleus. This implies that excitation energy and unpaired nucleons are predominantly transferred to the heavy fragment. Our results indicate that the time for transfer of excitation energy and nucleons is shorter than the saddle-to-scission time.
\end{abstract}

\section{Introduction}

The most prominent manifestation of pairing correlations in nuclear fission is the enhanced production of even-Z elements in low-energy fission of an even-Z compound nucleus. The left of Figure 1 shows the $\mathrm{Z}$ distribution observed in the fission of ${ }^{229} \mathrm{Th}$, which was produced as a secondary beam from 1 A GeV ${ }^{238} \mathrm{U}$ projectiles and was excited in the Coulomb field of lead target atoms slightly above the fission barrier with a width of about $5 \mathrm{MeV}$ (FWHM) [1]. The global shape of the data from Figure 1 can be described by three humps, one at symmetry and two at asymmetry. The even-odd effect in fissionfragment yields is the fine structure that is superimposed to the gross shape of the yields. The humps result from the shape of the potential as a function of mass (or charge) asymmetry as given by the liquid-drop with influence of shell effects. It was proposed in ref. [2] to quantify the even-odd staggering by the local even-odd effect $\delta_{\mathrm{p}}(Z)$, which corresponds to the third differences of the logarithm of the yields $Y$ :

$$
\delta_{\mathrm{p}}(Z)=\frac{1}{8}(-1)^{Z}[\ln Y(Z+3)-\ln Y(Z)-3(\ln Y(Z+2)-\ln Y(Z+1))] .
$$

Thus, $\delta_{\mathrm{p}}(Z)$ describes the local deviation from a Gaussian-like distribution. $\delta_{\mathrm{p}}(Z)$ filters out from the yields the variations that extend over a significant number of charges and are caused by the global shape of the potential energy. We can consider two curves; one links the logarithms of the yields of neighboring even-Z fragments $\ln Y_{\text {even }}(Z)$, and the other connects the logarithms of the yields of neighboring odd- $Z$ fragments $\ln Y_{\text {odd }}(Z)$. These two curves are continuous functions (if the yields $Y_{\text {even }}$ and $Y_{\text {odd }}$ follow a Gaussian shape the curves are parabolas) and can be evaluated for any value of $Z$. As explained by

\footnotetext{
ae-mail: jurado@cenbg.in2p3.fr
}

This is an Open Access article distributed under the terms of the Creative Commons Attribution License 2.0, which permits unrestricted use, distribution, and reproduction in any medium, provided the original work is properly cited. 

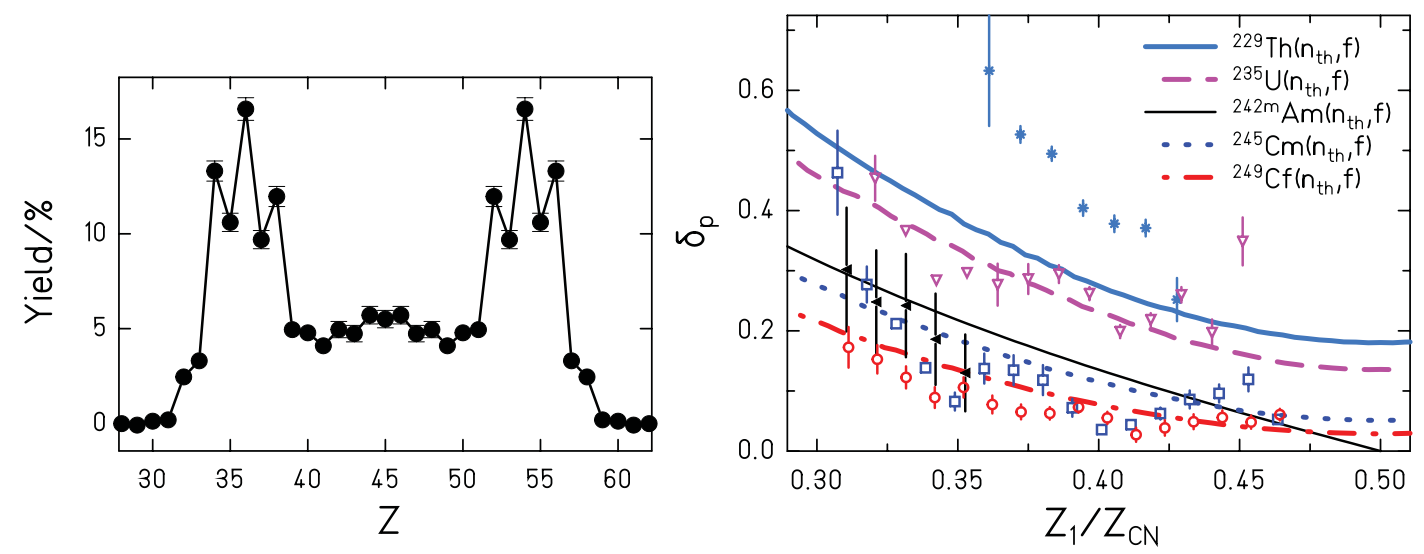

Figure 1. (Left) element distribution observed in the electromagnetic-induced fission of ${ }^{229} \mathrm{Th}$ [1]. (Right) local even-odd effect as a function of asymmetry, parameterized as the ratio of the charge of the light fragment $Z_{1}$ and the charge of the fissioning nucleus $Z_{C N}$. The symbols represent the experimental data which are taken from the compilation shown in ref. [3]. The lines correspond to the statistical calculation developed in this work.

Tracy et al. [2], $\delta_{\mathrm{p}}(Z)$ equals half the distance between the two curves. That is:

$$
\delta_{\mathrm{p}}(Z)=0.5\left(\ln Y_{\text {even }}(Z)-\ln Y_{\text {odd }}(Z)\right) .
$$

Several systematic features have been established experimentally regarding $\delta_{\mathrm{p}}(Z)$ [3]. The right part of Figure 1 shows experimental data on $\delta_{\mathrm{p}}(Z)$ as a function of charge asymmetry for different fissioning nuclei measured at ILL Grenoble where fission was induced by thermal neutrons. These data and the systematics compiled in [3] clearly illustrate several features:

(i) The amplitude of $\delta_{\mathrm{p}}$ decreases with increasing mass of the fissioning system.

(ii) For a given fissioning nucleus $\delta_{\mathrm{p}}$ increases with asymmetry.

(iii) Also odd-Z fissioning systems like ${ }^{243} \mathrm{Am}$ show an even-odd effect at large asymmetry whose magnitude is about the same as for even- $Z$ systems of comparable mass.

As described in [3], presently there is no consistent explanation for all these features. In this contribution, we present a model for the even-odd effect that is based on statistical mechanics.

\section{Model based on statistical mechanics}

We assume statistical equilibrium among the different types of degrees of freedom (intrinsic and collective) in the region around the outer saddle point. This implies that the available energy is equally shared between the different degrees of freedom. Except for very low excitation energies, e.g. below the pairing gap in even-even nuclei, most of the available states are intrinsic excitations. Thus, most of the excitation energy available above the outer saddle is stored in intrinsic excitations. That means that the intrinsic excitation energy at the outer saddle is essentially equal to the total energy of the nucleus minus the height of the outer fission barrier. The intrinsic excitation energy grows on the way from saddle to scission because part of the potential-energy release is dissipated into intrinsic excitations. Two-centre shell-model calculations shown in Figure 12 of [4] show that there are many level crossings on the first section behind the outer saddle. Afterwards, the single-particle levels change only little. Level crossings lead to intrinsic excitations [5]. This implies that the additional excitation energy is mostly dissipated in the vicinity of the outer saddle. The dissipated energy increases with the mass of the fissioning nucleus because the fission barrier is located at smaller deformations and the range with a high number of level crossings is extended. Additional intrinsic excitation may appear at neck rupture. 
Theoretical investigations of the gradual transition from the mononucleus regime to the dinuclear system concerning shell effects [4], pairing correlations [6] and congruence energy [7] show that the properties of the individual fission fragments are already very well established in the vicinity of the outer saddle. Therefore, close to the outer saddle the fissioning system consists of two well-defined nuclei in contact through the neck and a total amount of excitation energy $E_{t o t}$ that is equal to the intrinsic excitation energy above the outer saddle plus the energy acquired by dissipation on the first section behind the outer saddle. Intrinsic excitations are expected to be homogeneously distributed within the nuclear volume. This is likely to hold also in the transition from a mononuclear to a dinuclear system that takes place very rapidly near the outer saddle [4]. Therefore, a reasonable assumption is that $E_{t o t}$ is initially shared among the fragments according to the ratio of their masses.

The intrinsic excitation energy will be then distributed among the two nascent fragments according to the statistical weights of the available states. The partition of $E_{t o t}$ among the two fragments at statistical equilibrium is thus ruled by the level densities of both fragments. It was shown that the constant-temperature behaviour of the nuclear level density in the low-energy regime leads to an energysorting process if two nuclei are in thermal contact. In the absence of strong shell effects, the light fragment will transfer essentially all its excitation energy to the heavy one [8]. This process of energy sorting explains why an increase of the initial excitation energy leads to an increase of the number of emitted neutrons from the heavy fission fragment, only.

Nucleon exchange between the fragments establishes also an equilibrium between even-even, evenodd, odd-even, and odd-odd light and heavy nascent fragments with a restriction on the gross mass asymmetry given by the bottom of the potential in the fission valleys and on the total intrinsic excitation energy $E_{t o t}$. To obtain the probability of populating a given configuration at statistical equilibrium and derive the local even-odd effect $\delta_{\mathrm{p}}$ we have to consider the level densities of neighboring even-even, even-odd, odd-even or odd-odd nuclei in an absolute energy scale. However, as said above, the quantity $\delta_{\mathrm{p}}$ (Eq. (1)) filters out the slowly-varying components of the yields. Therefore, we have to use level densities where these effects are filtered out as well. As illustrated when discussing the meaning of $\delta_{\mathrm{p}}$, we can consider a smooth surface (in $N$ and $Z$ space) that connects the yields of odd-odd fragments. This surface can be associated with the potential energy in the fission valley corresponding to oddodd pre-fragments. The potential surface of odd-mass nuclei is at an energy $-\Delta$ ( $\Delta$ being the pairing gap $\approx 12 / \sqrt{A}$ ) with respect to the surface of odd-odd nuclei, and the potential surface of even-even nuclei is at an energy of $-2 \Delta$. The required filtering of the level densities can thus be obtained by placing the level densities in an excitation-energy scale that is reduced by $2 \Delta$ for even-even nuclei, by $\Delta$ for even-odd and odd-even nuclei and is left unchanged for odd-odd nuclei. We have done this on Figure 2 with the experimental level densities determined by the Oslo method [9] for various even-even, even-odd, odd-even and odd-odd nuclei located in two distinct mass regions around A = 165 and 45 . These two groups of nuclei are representative of the complementary fission fragments produced in very asymmetric fission. Within the heavy group, the level densities of neighboring even-even and even-odd nuclei are almost identical. Sizeable differences appear only in the energy interval $-2 \Delta_{2}<E<-\Delta_{2}$, where only even-even nuclei have states. For the light-mass group, the level densities converge well at positive energies. Significant fluctuations are present at negative energies due to structure effects since these nuclei are relatively light and located between the $Z=20$ and the $Z=28$ shell closures. Figure 2 clearly shows that the logarithmic slope of the level densities is nearly constant and that the logarithmic slope of the heavy group is much larger than the one of the light group.

The total amount of possible configurations with particular values of $Z_{1}$ and $Z_{2}$ is directly related to the integral of the total level density for that particular split over the excitation energy. This integral runs over the excitation energy of one fragment $E_{i}$, with the condition that $E_{1}+E_{2}=E_{\text {tot }}$, and reflects the freedom of the system in the division of excitation energy. The total level density is given by the product of the level densities of the two fragments $\rho_{1}\left(E_{1}\right) \rho_{2}\left(E_{2}\right)$. The most probable configuration is the one that provides the highest total level density. Starting from a situation where $E_{1}$ and $E_{2}$ are in the 


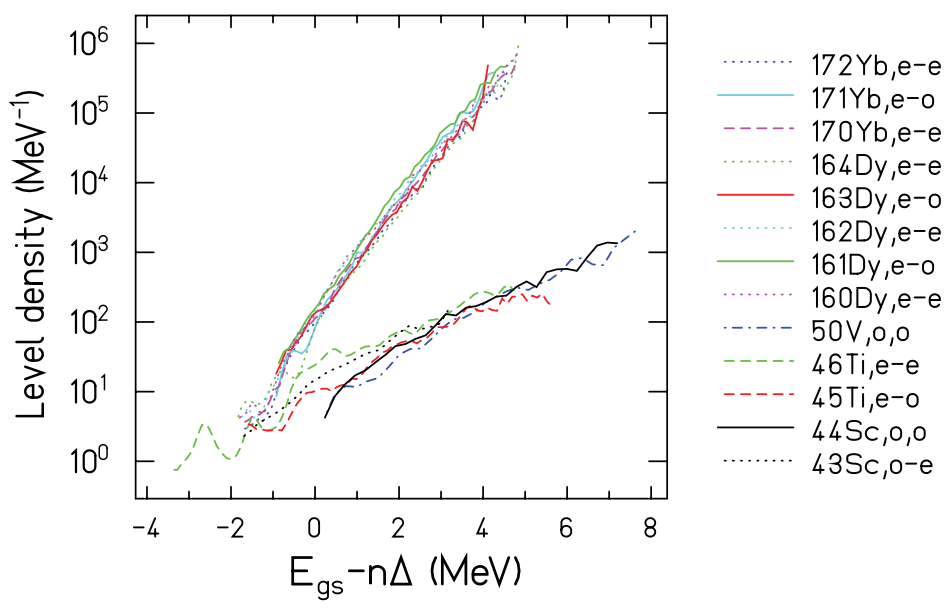

Figure 2. Experimental level densities of various nuclei [9]. The excitation energy is reduced by $2 \Delta$ for even-even (e-e) nuclei, by $\Delta$ for even-odd (e-o) or odd-even (o-e) nuclei and left unchanged for odd-odd (o-o) nuclei.

range of energy shown in Figure 2, due to the very different logarithmic slopes of the level densities for the heavy and the light fragment group, the most favorable configurations are those that minimize the excitation energy of the light fragment. In other words, energy sorting takes place [8]. Only at the end of the energy sorting, when $E_{1} \approx 0$ and $E_{2} \approx E_{\text {tot }}$, the advantage of forming an even-odd or an even-even light fragment becomes apparent because only for these configurations there are states available. If an even-even light fragment in the ground state is formed, instead of an odd-odd one, the energy in the heavy fragment increases to $E_{t o t}+2 \Delta_{1}$, which for the nuclei considered in Figure 2 corresponds to an increase of the level density of the heavy fragment of more than three orders of magnitude. It becomes clear that configurations with fully paired light fragments are strongly favored for very asymmetric fission. Because the logarithmic slope of the constant-temperature level densities is roughly proportional to $\mathrm{A}^{2 / 3}$ [10], for less asymmetric splits the logarithmic slopes of the level densities of the two fragments will become closer. Therefore, the probability to populate the ground states of even-even or even-odd light fragments decreases with decreasing asymmetry.

For an even-even fissioning nucleus, the number of configurations with $Z_{1}$ even is given by:

$$
N_{Z_{1}=e}^{e e}\left(Z_{1}\right)=\int_{-2 \Delta_{1}}^{E_{t o t}+2 \Delta_{2}} \rho_{1}\left(E_{1}\right)_{(e e)} \rho_{2}\left(E_{t o t}-E_{1}\right)_{(e e)} d E_{1}+\int_{-\Delta_{1}}^{E_{\text {tot }}+\Delta_{2}} \rho_{1}\left(E_{1}\right)_{(e o)} \rho_{2}\left(E_{t o t}-E_{1}\right)_{(e o)} d E_{1}
$$

where $\rho_{i}\left(E_{i}\right)_{(e e)}$ are the level densities of representative even-even nuclei with mass close to $A_{1}$ or $A_{2}$, and $\rho_{i}\left(E_{i}\right)_{(e o)}$ are the level densities of representative even-odd nuclei with mass close to $A_{1}$ or $A_{2}$. The number of configurations with $Z_{1}$ odd is:

$$
N_{Z_{1}=o}^{e e}\left(Z_{1}\right)=\int_{-\Delta_{1}}^{E_{t o t}+\Delta_{2}} \rho_{1}\left(E_{1}\right)_{(o e)} \rho_{2}\left(E_{t o t}-E_{1}\right)_{(o e)} d E_{1}+\int_{0}^{E_{t o t}} \rho_{1}\left(E_{1}\right)_{(o o)} \rho_{2}\left(E_{t o t}-E_{1}\right)_{(o o)} d E_{1}
$$

where $\rho_{i}\left(E_{i}\right)_{(o e)}$ is the level density of a representative odd-even nucleus with mass close to $A_{1}$ or $A_{2}$, and $\rho_{i}\left(E_{i}\right)_{(o o)}$ is the level density of a representative odd-odd nucleus with mass close to $A_{1}$ or $A_{2}$. The yield for even- $Z_{1}$ nuclei is $Y_{Z_{1}=e}^{e e}\left(Z_{1}\right)=N_{Z_{1}=e}^{e e}\left(Z_{1}\right) / N_{\text {tot }}^{e e}\left(Z_{1}\right)$ with $N_{\text {tot }}^{e e}\left(Z_{1}\right)=N_{Z_{1}=e}^{e e}\left(Z_{1}\right)+N_{Z_{1}=o}^{e e}\left(Z_{1}\right)$. 
Similarly, for an odd-even fissioning nucleus, we have:

$$
N_{Z_{1}=e}^{o e}\left(Z_{1}\right)=\int_{-2 \Delta_{1}}^{E_{t o t}+\Delta_{2}} \rho_{1}\left(E_{1}\right)_{(e e)} \rho_{2}\left(E_{t o t}-E_{1}\right)_{(o e)} d E_{1}+\int_{-\Delta_{1}}^{E_{t o t}} \rho_{1}\left(E_{1}\right)_{(e o)} \rho_{2}\left(E_{t o t}-E_{1}\right)_{(o o)} d E_{1}
$$

and

$$
N_{Z_{1}=o}^{o e}\left(Z_{1}\right)=\int_{-\Delta_{1}}^{E_{t o t}+2 \Delta_{2}} \rho_{1}\left(E_{1}\right)_{(o e)} \rho_{2}\left(E_{t o t}-E_{1}\right)_{(e e)} d E_{1}+\int_{0}^{E_{t o t}+\Delta_{2}} \rho_{1}\left(E_{1}\right)_{(o o)} \rho_{2}\left(E_{t o t}-E_{1}\right)_{(e o)} d E_{1} .
$$

In the particular energy scale used in Eqs. (3)-(6), the level densities of neighboring even-even, odd-A and odd-odd nuclei are very similar for positive excitation energies (see Fig. 2). Therefore, the difference between the number of configurations $N_{Z_{1}=e}$ and $N_{Z_{1}=o}$ is mainly given by the different limits of the integrals. For even-even fissioning nuclei, Eqs. (2) and (3) show that $N_{Z_{1}=e}^{e e}>N_{Z_{1}=o}^{e e}$ for every charge split $Z_{1}$ and $Z_{2}$ and the even-odd effect will always be different from zero. In contrast, for odd-even fissioning nuclei $N_{Z_{1}=e}^{o e} \approx N_{Z_{1}=o}^{o e}$ close to symmetry because $\Delta_{1} \approx \Delta_{2}$. $N_{Z_{1}=e}^{o e}$ starts to be larger than $N_{Z_{1}=o}^{o e}$ as we move to more asymmetric splits because $\Delta_{1}>\Delta_{2}$.

In practice one cannot always find experimental level densities representative of even-even, oddA or odd-odd nuclei for each value of $Z_{1}$ and $Z_{2}$ covered by the fission yields. However, given the similarity between the experimental level densities of neighboring nuclei, we have replaced in Eqs. (3)(6) the representative level densities by the level densities $\rho_{i}$ of the two fission fragments considered, namely $A_{1}, Z_{1}$ and $A_{2}, Z_{2}$. The level densities $\rho_{i}$ are obtained using a composite formula with a constant-temperature description at low excitation energies and a shifted Fermi-gas description above. In order to include the effects of pairing correlations on the nuclear level density in a realistic way, the recommended energy shift of the Fermi-gas part of the Gilbert-Cameron composite formula [11] is increased by $2 \mathrm{MeV}$. Consequently, in our composite formula the transition from the constanttemperature to the Fermi-gas regime occurs at energies of about $8-9 \mathrm{MeV}$ which are higher than the transition energies of the broadly used Gilbert-Cameron formula [12]. For fragment energies larger than the transition energy, the level densities of the fragments follow the Fermi-gas description. Due to the softer logarithmic slope of the Fermi-gas level density, the relative statistical weight of configurations with a fully-paired light fragment is less important than in the constant-temperature regime. Therefore, the transition from the constant-temperature to the Fermi-gas regime that may occur when $E_{t o t}$ increases will lead to a considerable decrease of $\delta_{\mathrm{p}}$. In our description, we assume that the level densities follow the constant-temperature behavior down to $-\Delta$ for odd-mass and down to $-2 \Delta$ for even-even nuclei. As shown in Figure 2, this is rather reasonable for most of the fission fragments. The reason is the presence of collective levels and the energy resolution of the measurements. The latter leads to a kind of averaging that can be justified by the fluctuations of the energy exchange [13].

The results of our calculation are compared with the thermal-neutron-induced data of several nuclei on the right part of Figure 1. Except for ${ }^{230} \mathrm{Th}$, the increase of $\delta_{\mathrm{p}}$ with asymmetry and the decrease of $\delta_{\mathrm{p}}$ with increasing mass of the fissioning nucleus are fairly well reproduced. For ${ }^{236} \mathrm{U}$, the data point that is closest to symmetry is appreciably higher than the calculation. This effect may be associated to the influence of the $Z=50$ shell in the complementary fragment, which is known to enhance the yield of tin isotopes. Our statistical calculation is also in good agreement with the data for the odd$\mathrm{Z}$ fissioning nucleus ${ }^{243} \mathrm{Am}$. Unfortunately, these data do not reach symmetry where our calculations predict no even-odd effect. In our calculation, we consider that $40 \%$ of the potential energy difference from saddle to scission [14] is dissipated for all fissioning nuclei. When $E_{\text {tot }}$ is small, as is the case for ${ }^{230} \mathrm{Th}, \delta_{\mathrm{p}}$ varies very rapidly with $E_{t o t}$. Therefore, ${ }^{230} \mathrm{Th}$ is particularly sensitive to the uncertainties on the dissipated energy. The disagreement found for ${ }^{230} \mathrm{Th}$ may be caused by the neglect of fluctuations in the dissipated energy acquired on the way from saddle to scission that are due to shape variations. In 
fact, for a great part of the fission events the available energy may be so low that they reach the scission point in a completely paired configuration due to the threshold character of the first quasi-particle excitation.

The formation of a fully-paired light fragment (a light fragment with no quasi-particle excitations) takes some time. This time is the sum of the time needed for the light fragment to transfer its energy to the heavier one and the time to transfer one or two unpaired nucleons through the neck. If this time is longer than the saddle to scission time, the even-odd effect will be reduced. Therefore, the agreement between the experimental data and the statistical calculation shown in Figure 1 indicates that the time to transfer few $\mathrm{MeV}$ of excitation energy and few nucleons between the fragments is shorter than the saddle to scission time. In principle, the energy dissipated at neck rupture adds up to the excitation energies of the fragments in fixed proportions, given by the geometry of the necking configuration. However, we expect that this additional excitation energy does not influence the evenodd effect as predicted by our model because there is not enough time at scission to distribute this excitation energy according to statistical equilibrium and to further modify the number of nucleons of the fragments.

\section{Conclusion}

We have shown that the general dependence of the even-odd effect in fission-fragment yields with the mass of the fissioning system and with the mass asymmetry of the fragments can be explained by the preferential population of even-even light fragments with no quasi-particle excitations. This implies that excitation energy and unpaired nucleons are predominantly found in the heavy fragment. Thus, the even-odd effect in fission-fragment yields gives a second strong evidence for the importance of the energy-sorting mechanism in low-energy fission, which is fully consistent with the excitation-energy dependence of the prompt-fission neutron yields [8].

The fairly good agreement between our model based on statistical mechanics and the experimental data means that the dynamical saddle-to-scission time is long enough for the intrinsic excitations of the nascent fragments to reach statistical equilibrium before scission. This is an interesting finding that puts constraints on the time scales of the shape evolution and of heat transport in large-amplitude collective motions under the influence of pairing correlations, which represents still a considerable challenge.

To further test our calculations, new experimental data are needed on the variation of the even-odd effect with asymmetry and excitation energy for different even- and odd-Z fissioning nuclei.

This work was supported by the European Commission within the Sixth Framework Programme through EFNUDAT (project no. 036434) and within the Seventh Framework Programme through Fission-2010-ERINDA (project no. 269499).

\section{References}

[1] K.-H. Schmidt et al., Nucl. Phys. A 665, 221 (2000)

[2] B. L. Tracy et al., Phys. Rev. C 5, 222 (1972)

[3] M. Caamano, F. Rejmund, K.-H. Schmidt, J. Phys. G: Nucl. Part. Phys. 38, 035101 (2011)

[4] U. Mosel and H. W. Schmitt, Nucl. Phys. A 165, 73 (1971)

[5] M. Mirea, Phys. Lett. B 680 (2009) 316

[6] H. J. Krappe, Int. J. Mod. Phys. E 16, 396 (2007)

[7] W.D. Myers and W. J. Swiatecki, Nucl. Phys. A 612, 249 (1997)

[8] K.-H. Schmidt, B. Jurado, Phys. Rev. Lett. 104, 212501 (2010); Phys. Rev. C 83, 061601 (2011) 
[9] A. C. Larsen et al., Phys. Rev. C 83, 034315 (2011)

[10] T. von Egidy, D. Bucurescu, Phys. Rev. C 72, 044311 (2005)

[11] R. Capote et al., Nucl. Data Sheets 110, 3107 (2009)

[12] K.-H. Schmidt, B. Jurado, Phys. Rev. C 86, 044322 (2012)

[13] K.-H. Schmidt, B. Jurado, Phys. Rev. C 83, 014607 (2011)

[14] M. Asghar and R. W. Hasse, J. Phys. Colloques 45, C6-455 (1984) 